\title{
Mégaprojets et développement territorial : cas des industries extractives en Afrique
}

\author{
Christelle Nzoyem, Thierno Diallo, Christophe Leyrie et Julien Bousquet ${ }^{1}$
}

\section{INTRODUCTION}

\section{Dans les pays en développement riches en ressources naturelles, les populations semblent confrontées à une redoutable agression de la part d'exploitations minières à grande échelle dont le nombre et le gigantisme ne cesse de croître depuis la fin des années 1990.}

Depuis les deux dernières décennies, le secteur des industries extractives tient une place importante dans les études et les débats sur les questions relatives au développement. Ceci traduit l'intérêt grandissant porté à l'exploitation minière en tant qu'aspect essentiel du processus de développement. Dans les pays en développement riches en ressources naturelles, les populations semblent confrontées à une redoutable agression de la part d'exploitations minières à grande échelle dont le nombre et le gigantisme ne cesse de croître depuis la fin des années 1990. L'épuisement des ressources de minerais de qualité se traduit par un déploiement croissant des compagnies minières qui, moyennant de capitaux importants, de techniques modernes permettant une grande extraction, procurent aux pays hôtes des bénéfices bien supérieurs à ceux obtenus dans la plupart des industries, cependant celles-ci laissent généralement derrière elles un passif environnement, social et économique irréparable. De ce fait, ces entreprises sont exposées, plus encore que dans tout autre secteur, à des allégations en termes de violation des droits de l'homme, de relations avec les communautés locales et de compensation.

La richesse minérale de l'Afrique ne laisse aucun doute. Même si ce fait est connu, certains chiffres méritent d'être rappelés. Selon le dernier rapport de la United States Geological Survey ${ }^{2}$, la contribution du continent africain en pourcentage de la production mondiale en 2005 était pour le chrome $44 \%$; le cobalt $57 \%$; l'or $21 \%$; le manganèse $39 \%$; le diamant $46 \%$; et le phosphate $31 \%$. Pour ce qui est des réserves, selon le Programme des Nations Unies pour l'environnement (PNUE), l'Afrique contiendrait environ $30 \%$ des réserves mondiales en minéraux connues mondialement, incluant $40 \%$ de l'or mondial, $60 \%$ du cobalt et $90 \%$ des réserves mondiales de minéraux du groupe platine ${ }^{3}$.

Fort de cette réalité, il est souvent affirmé que le secteur minier devra servir de «moteur de la croissance » et d'instrument de réduction de la pauvreté. Le rôle du secteur comme outil de réduction de la pauvreté est maintes fois réaffirmé dans les Documents Stratégiques de Réduction de la Pauvreté. Dans le cadre de nombreuses réflexions menées sur l'implantation des mégaprojets en Afrique, le moment semble propice pour identifier quelques enjeux liés à la contribution du secteur minier au développement économique et social des pays et régions concernés. Dans cet article, notre principale contribution est de générer des idées susceptibles de faciliter la compréhension de la prolifération des projets miniers et énergétiques en Afrique et de comprendre leurs impacts sur le développement territorial. L'article tente d'éclaircir si le bilan de la contribution passée du secteur minier au développement en Afrique. Pour cela nous faisons le point sur la question des grands projets en analysant les rôles, défis, contributions et retombées sociales et environnementales de ces grands projets.

Dans le cadre de nombreuses réflexions menées sur l'implantation des mégaprojets en Afrique, le moment semble propice pour identifier quelques enjeux liés à la contribution du secteur minier au développement économique et social des pays et régions concernés. 


\section{IMPACTS ENVIRONNEMENTAUX, ÉCONOMIQUES, SOCIAUX ET POLITIQUES}

Certains experts ont noté que les pays riches en minéraux ont obtenu des résultats inférieurs aux pays moins bien dotés au regard de divers indicateurs de la performance économique, sociale et politique. D'autres ont fait valoir que les ressources minérales, si elles sont bien gérées, constituent une source potentielle de croissance et de développement. Toutefois, les opérations d'extraction, quel que soit celui qui les réalise, s'accompagnent de coûts.

\section{Si l'on veut que les revenus des industries extractives contribuent à accélérer le développement, il est indispensable de déployer des efforts considérables, mais surtout de relever des défis afin de traiter ces questions.}

Sur le plan environnemental, elles peuvent contribuer à la dégradation de l'environnement si l'extraction tient uniquement à leur présence, mais d'autre part elles peuvent atténuer les conséquences sur l'environnement en utilisant des technologies de production plus modernes et en appliquant et en diffusant des normes de gestion de l'environnement plus déterminées de façon non négligeable par la réglementation locale et la capacité du gouvernement du pays hôte à l'appliquer ${ }^{4}$.

Ces dernières années, les grands exploitants du secteur de l'extraction minière comme du secteur du pétrole et du gaz ont pris de plus en plus conscience de la question environnementale. S'il y a encore des accidents et si de mauvaises pratiques persistent, la situation en la matière s'est d'une manière générale améliorée au cours des dix dernières années environ, bien qu'à un rythme variable selon les entreprises. Par exemple, les entreprises minières basées dans des pays où la législation environnementale ne fait que commencer à se développer seront relativement moins à même de gérer les conséquences environnementales de leurs projets à l'étranger que celles basées dans des pays où la législation est plus développée et les normes plus rigoureuses.
Sur le plan économique, la présence des firmes énergétiques a un prix; elles gardent pour elles une part significative des recettes et rapatrient une certaine partie de leurs bénéfices, ce qui diminue d'autant la création de valeurs pour le pays. De plus, celles-ci capturent la valeur créée localement sous forme d'emplois et de salaires, d'achats et de recettes publiques et n'ont donc de ce point de vue qu'un faible impact, du moins au niveau macroéconomique. Cela est particulièrement vrai, étant donné que celles-ci ont tendance à utiliser des technologies et des procédés à plus forte intensité de capital que les entreprises locales. De plus, les filiales étran-gères auront plutôt tendance à faire appel à des fournisseurs étrangers. Dans les pays à faible revenu, l'absence de fournisseurs et de main-d'œuvre qualifiés peut également limiter le recours à la production locale ainsi que les possibilités de transformation en aval.

Sur le plan social, la santé et la sécurité ont toujours posé problème, notamment, dans le secteur de l'extraction artisanale des pays en développement. Les conflits entre exploitants miniers et les communautés locales, l'afflux de migrants à la recherche d'un emploi et des questions connexes peuvent également être préoccupants.

Encore plus que dans d'autres secteurs industriels, les investissements dans le secteur extractif peuvent avoir des conséquences politiques considérables, qui dépendent dans une large mesure de la situation du pays d'accueil. Les impacts politiques négatifs ont été observés principalement dans les pays pauvres disposant d'importantes ressources minières, mais dont les institutions sont faibles. Les problèmes sont fréquemment associés au faible développement des cadres de gouvernance et à la faiblesse des capacités institutionnelles pour ce qui est de formuler et d'appliquer les lois et règlements.

Si l'on veut que les revenus des industries extractives contribuent à accélérer le développement, il est indispensable de déployer des efforts considérables, mais surtout de relever des défis afin de traiter ces questions. 


\section{MÉGAPROJETS : LES DÉFIS PARTICULIERS À RELEVER}

\section{RSE : un défi de gestion particulier à relever par les grands projets}

La prise en compte de l'intégralité des impacts directs et indirects (les impacts multiplicateurs et de diversification dépassant le champ du secteur minier) est encore embryonnaire.

L'activité minière est considérée comme moteur du développement macroéconomique de plusieurs États en ceci qu'elle induit divers effets économiques potentiels. S'il est vrai que les entreprises minières nationales et transnationales $^{5}$ sont conscientes des risques et de l'impact que leur projet va vraisemblablement créer, force est alors de constater que la prise en compte de l'intégralité des impacts directs et indirects (les impacts multiplicateurs et de diversification dépassant le champ du secteur minier) est encore embryonnaire. Toutefois, les entreprises minières sont peu à peu sensibilisées et comprennent comment gérer et équilibrer leurs besoins économiques avec les considérations environnementales et les traditions culturelles des peuples des régions dans lesquelles elles opèrent. En effet, la question de la Responsabilité Sociétale des Entreprises (RSE) prend une dimension particulière lorsqu'elle porte sur des acteurs aussi spécifiques que les Industries Extractives et devient alors un réel défi de gestion à relever.

En effet, les communautés subissent divers coûts sociaux qui ne compensent malheureusement pas les pertes contractées. Prenons pour exemple, l'utilisation et la gestion des terres, les déplacements des populations (y compris les autochtones), les privations des terres et des moyens d'existence, etc. Le cas le plus concret que nous pouvons décrire est celui relatif à la construction du pipeline Tchad - Cameroun où les terres et les biens de la population locale ont été expropriés; avec une indemnisation dans certains cas, mais à des taux considérés comme inéquitables par la communauté ${ }^{6}$.

Ainsi, porter une attention systématique aux intérêts et préoccupations des individus et groupes affectés par l'entreprise devient un défi pour les industries extractives. Face à ce défi, elles semblent de plus en plus orientées vers l'investissement responsable et s'attachent à répondre aux besoins des communautés locales. La démarche au cœur de ce défi est incontestablement la stratégie RSE de l'entreprise qui exploite.

\section{Les défis économiques}

La valeur qu'un pays peut chercher à mobiliser au profit de l'économie locale peut être soit directe, sous la forme d'emplois, de bénéfices et d'impôts, soit indirecte, par l'acquisition de biens et services. Ici encore, les possibilités dépendent du mode d'organisation de l'activité minière ainsi que de la nature des minéraux extraits et du niveau des capacités locales.

Dans les pays en développement, il est en général difficile d'acquérir sur place les intrants hautement spécialisés servant à l'exploration et à l'extraction de minéraux; souvent, les achats locaux portent seulement sur des activités comme la restauration, le nettoyage et, dans certains cas, les services de construction. Cela étant, ce sont sans doute les recettes fiscales et les bénéfices tirés des activités extractives qui apportent la part de valeur la plus importante à l'économie d'un pays en développement. De ce fait, le défi à relever concerne l'usage qui est fait des revenus tirés de l'extraction de minéraux, point d'importance décisive dans la perspective du développement et centre d'intérêt principal de l'action gouvernementale.

\section{Le défi de la gouvernance}

La capacité d'un pays à répondre avec succès à tous les défis importants, économiques, environnementaux, sociaux et politiques, dépend dans une large mesure de son système de gouvernance.

La capacité d'un pays à répondre avec succès à tous les défis importants, économiques, environnementaux, sociaux et politiques, dépend dans une large mesure de son système de gouvernance. 
L'ONU a défini la gouvernance comme «le fait d'exercer l'autorité économique, politique et administrative pour gérer les affaires d'un pays à tous les niveaux ». Elle se caractérise par :

«... la participation, la transparence et la responsabilité. Elle est également efficace, et équitable, mais aussi propice à la légalité. En bonne gouvernance, les priorités politiques, sociales et économiques sont fondées sur un large consensus au sein de la société et les voix des plus pauvres et des plus vulnérables sont entendues par les décideurs chargés d'affecter les ressources prévues pour le développement ${ }^{7}$

L'absence d'un cadre de gouvernance bien configuré accroît le risque que les avantages potentiels des activités extractives ne voient pas le jour, que les systèmes fiscaux aboutissent à une répartition inégale des recettes, que l'absence d'une stratégie de développement cohérente et concertée conduise à une mauvaise utilisation de ces dernières, que les populations locales vivent dans la déception et que surviennent des dommages à l'environnement, des risques sanitaires et des conflits. La qualité des institutions avant la découverte de ressources minérales et la capacité d'un pays à réguler, contrôler et discipliner les activités des industries extractives sont des éléments essentiels à prendre en compte. Un autre défi à relever de la part des gouvernements pour faire face aux impératifs souvent difficiles à concilier du fait de l'introduction des firmes extractives est de retrouver son rôle de médiation des relations socio-économiques au lieu de laisser les entreprises privées de plus en plus sujettes à répondre à des demandes sociales des communautés concernées.

\section{MÉGAPROJETS MINIERS : UN FILON À RENTABILISER POUR LE DÉVELOPPEMENT DES COMMUNAUTÉS}

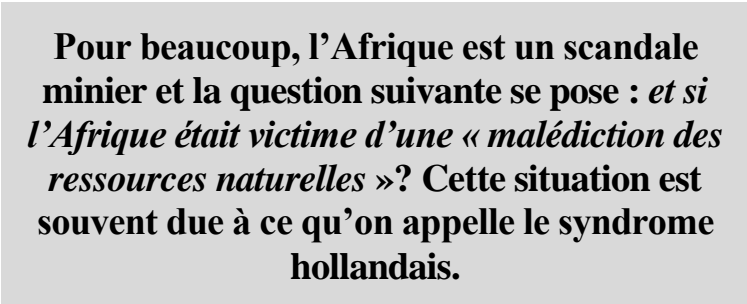

Pour beaucoup, l'Afrique est un scandale minier et la question suivante se pose : et si l'Afrique était victime d'une "malédiction des ressources naturelles »? Cette situation est souvent due à ce qu'on appelle le syndrome hollandais ou la malédiction des ressources naturelles et fait que les revenus élevés générés par les minerais limitent la diversification des structures, ce qui empêche les économies de transformer l'abondance des ressources en croissance durable qui permet d'améliorer les conditions de vie des communautés. Théoriquement, l'exploitation minière devrait favoriser le développement d'activités dérivées qui fournissent aux sociétés minières une gamme d'intrants. Ces activités, bien que générées par l'exploitation minière, peuvent trouver des débouchés dans d'autres secteurs de développement du pays, accélérant ainsi la croissance.
Or, en raison d'une gouvernance médiocre, les recettes tirées des activités extractives ont souvent été gaspillées plutôt que d'être investies de manière à favoriser le développement durable. Les systèmes de gouvernance et les capacités institutionnelles doivent donc être renforcés et la richesse minérale être utilisée pour investir dans le renforcement des connaissances en faveur de l'innovation économique et dans la formation de capital humain, social et matériel, en particulier sur le plan des infrastructures ${ }^{8}$. La présence de ressources minières est un facteur de développement et peut contribuer à la réduction de la pauvreté. D'ailleurs, certains pays développés actuels ainsi qu'un certain nombre de pays en développement en ont tiré parti avec succès pour accélérer leur développement.

Néanmoins, l'existence de ressources naturelles ne se traduit pas automatiquement par une accélération du développement, que les entreprises internationales participent ou non à leur exploitation. Ces dernières peuvent toutefois avoir une influence par leurs investissements intérieurs et leurs apports en capital et en technologie, de même que leur compétence en matière de gestion, 
peuvent permettre une forte augmentation de la croissance. Ainsi, le secteur minier va se positionner comme un moteur de la relance du développement qui, par ses effets d'entrainement, va permettre le développement local et celui des communautés riveraines des zones d'exploitation en particulier. La plupart des pays africains, ayant l'ambition d'accéder au rang de pays émergents doivent se doter d'un secteur industriel et minier fort. Rentabiliser ce filon revient à mettre en œuvre leur stratégie pour la croissance et l'emploi en intensifiant l'exploration, l'exploitation, la transformation et la commercialisation des ressources minières en attirant dans ces activités à haute intensité capitalistique et technologique les investisseurs.

Il ne fait aucun doute que les ressources minières peuvent favoriser une croissance et un développement généralisés, à condition

d'exploiter les possibilités offertes en matière de consolidation du secteur de ressources, grâce à l'optimisation du maillage des secteurs de l'économie nationale.

L'exploitation minière est par nature non durable, la durée de vie d'une mine étant limitée, et les ressources finissant par s'épuiser. Toutefois, on peut lui assurer une certaine durabilité, grâce aux liens (en amont, en aval et dérivés) qu'elle a avec d'autres secteurs de l'économie. Tel que défini par la Commission mondiale de l'environnement et du développement dans le rapport Brundtland intitulé « Notre avenir à tous ${ }^{9}$, le développement durable est « un développement qui répond aux besoins des générations actuelles sans compromettre la capacité des générations à venir de répondre aux leurs ». Cela dit, cette définition met l'accent sur l'équité entre générations. Si l'on considère les stratégies de développement fondées sur l'exploitation des ressources qui ont fait leurs preuves ailleurs, il ne fait aucun doute que les ressources

\section{CONCLUSION}

L'essor considérable de la demande mondiale de certains minerais depuis la fin $\mathrm{du}_{\mathrm{XX}}^{\mathrm{e}}$ siècle a conduit à la multiplication des grands projets miniers, concernant notamment des métaux très convoités et de plus en plus rares comme l'or. L'Afrique dispose d'immenses ressources natu- minières peuvent favoriser une croissance et un développement généralisés, à condition d'exploiter les possibilités offertes en matière de consolidation du secteur de ressources, grâce à l'optimisation du maillage des secteurs de l'économie nationale.

En effet, les connaissances scientifiques et la compétence des ingénieurs peuvent contribuer à accroître le volume des réserves prouvées, à améliorer les techniques d'extraction et de raffinage et à étendre le champ des utilisations finales et commerciales des produits. Un certain nombre de pays en développement aujourd'hui classés dans les catégories des pays à revenu élevé ou à revenu moyen supérieur (Afrique du Sud, Arabie saoudite, Botswana, Chili, Émirats arabes unis, Indonésie, Koweït, Malaisie et Venezuela, par exemple) sont parvenus, à des degrés divers, à tirer parti de leurs richesses naturelles pour faire progresser au moins certains aspects de leur développement (augmentation du revenu par habitant, réduction de la pauvreté et, dans certains cas, diversification économique). Les pays développés ont utilisé la plupart des minéraux extraits à l'intérieur de leurs frontières, les activités de transformation et les intrants locaux étant protégés par des coûts de transport élevés.

En outre, dans ce nouveau contexte global qui pourrait limiter la capacité relative des pays riches en minéraux à tirer parti de leurs richesses minérales, il leur faudra donc concevoir une stratégie globale de développement leur permettant d'exploiter ces richesses en vue non seulement d'améliorer leur situation présente, mais aussi d'assurer un développement durable dans l'intérêt des générations futures. Dans cette optique, l'un de leurs objectifs importants doit être de construire une économie diversifiée en investissant dans le capital humain, les infrastructures et les capacités de production ${ }^{10}$.

relles dont seule une fraction est actuellement exploitée par certaines firmes qui, conscientes de l'enjeu capital que constitue leur image environnementale, ont réussi, mieux que d'autres, à développer une stratégie, une communication et des actions permettant de rendre plus accep- 
tables leurs projets miniers, même quand ceux-ci ont un impact considérable sur l'environnement. En effet, elles s'efforcent de montrer les répercussions positives de leur activité en termes économiques et sociaux pour les populations locales. La majorité des grands projets miniers en cours de développement actuellement sont a priori suspectés par les gouvernements d'accueil d'avoir un impact très négatif et même dévastateur pour l'environnement et le développement socio-économique des populations locales. Aussi, pour que l'Afrique tire profit des avantages sociaux économiques inhérents à cette richesse, il est nécessaire de s'attaquer d'urgence à des questions telles que la bonne gestion de ces ressources et les impacts de leur exploitation. De ce fait, rappelons le principe 1 des règles de l'Initiative de Transparence pour les Industries Extractives (ITIE) qui stipule :

«Nous partageons la même conviction que l'exploitation prudente des richesses en ressources naturelles devrait constituer un moteur important pour une croissance économique qui contribue au développement durable et à la réduction de la pauvreté, mais qui, faute d'une bonne gestion, peut avoir des répercussions défavorables sur le plan économique et social. $»^{11}$

\section{BIBLIOGRAPHIE ET NOTES}

${ }^{1}$ Nzoyem Christelle est doctorante au programme de doctorat en management de projets. Thierno Diallo, Christophe Leyrie, et Julien Bousquet sont professeurs au département des sciences économiques et administratives à l'Université du Québec à Chicoutimi.

${ }^{2}$ Voir Yager, Thomas et al., (2005). The Mineral Industries of Africa, U.S. Geological Survey Minerals Yearbook.

${ }^{3}$ Voir Programme des Nations Unies pour l'environnement (PNUE) (2006). L'avenir de l'environnement en Afrique. Notre environnement, notre richesse. Nairobi : Division de l'Alerte Rapide et de l'Évaluation, p.87-99.

${ }^{4}$ Voir Conférence des Nations Unies sur le commerce et le développement (CNUCED). (2007). Rapport sur l'investissement dans le monde. Sociétés transnationales, industries extractives et développement.

${ }^{5}$ Le qualificatif transnational permet de traduire l'idée d'une stratégie à la fois infraétatique et transétatique qui caractérise ces acteurs.
${ }^{6}$ Centre pour l'Environnement et le développement (CED) (2010). Yaoundé.

${ }^{7}$ Programme des Nations Unies pour le Développement (PNUD) (2008).

${ }^{8}$ Conférence des Nations Unies sur le commerce et le développement (CNUCED). (2007). Rapport sur l'investissement dans le monde. Sociétés transnationales, industries extractives et développement

${ }^{9}$ World Commission on Environment and Developement (WCED) (1987).

${ }^{10}$ Organisation des Nations Unies (ONU), (2007). Rapport sur l'investissement dans le Monde. New York and Genève.

${ }^{11}$ Secrétariat International de l'ITIE, (2011). Règles de l'ITIE, Oslo. 